\title{
Malla de polipropileno monofilamento libre de tensión en la reparación concomitante de cistocele y rectocele
}

\author{
V. Solà Dalenz, J. Pardo Schanz, P. Ricci Arriola, E. Guiloff Fische \\ Unidad de Uroginecología y Cirugía Vaginal. Departamento de Ginecología y Obstetricia. \\ Clinica Las Condes. Santiago. Chile. \\ Actas Urol Esp 2005; 29 (10): 977-980
}

\section{RESUMEN}

\section{MALLA DE POLIPROPILENO MONOFILAMENTO LIBRE DE TENSIÓN EN LA REPARACIÓN CONCOMITANTE DE CISTOCELE Y RECTOCELE}

Objetivo: Presentamos nuestra experiencia en la corrección de cistocele y rectocele concomitante, con malla de polipropileno monofilamento (Gynemesh PS), con técnica libre de tensión transvaginal.

Método: Entre noviembre 2004 y enero 2005 se realizó corrección 7 pacientes, de la Unidad de Uroginecología y Cirugía Vaginal del Departamento de Ginecología y Obstetricia, de la Clínica Las Condes. Mediana de edad 54 años, peso $64 \mathrm{~kg}$. En cuatro pacientes además se asoció una tercera malla para corrección de incontinencia de orina de esfuerzo, con técnica de TVT-O.

Resultado: No se presentaron complicaciones intraoperatorias, ni en el postoperatorio inmediato ni tardío. No se observó hematoma, infección, ni exposición de la malla. Se obtuvo curación del cistocele y del rectocele en el $100 \%$ de las pacientes. No se han presentado a la fecha actual, completando 3 meses de observación 4 casos, 2 meses 2 casos, y 1 mes un caso.

Discusión: La utilización de mallas protésicas, de polipropileno monofilamento con macroporo en la corrección concomitante del cistocele y del rectocele, por vía transvaginal, con técnica libre de tensión, parece ser un procedimiento seguro y eficaz.

Palabras clave: Reparación de prolapso de órganos pélvicos. Cistocele. Rectocele. Malla de polipropileno. Libre de tensión.

\section{ABSTRACT \\ TENSION FREE MONOFILAMENT POLYPROPYLENE MESH IN CYSTOCELE AND RECTOCELE CONCOMITANT REPAIR}

Objective: We presented our experience in the concomitant correction of cystocele and rectocele with polypropylene monofilament mesh (Gynemesh PS), with transvaginal free tension technique.

Method: During 2004 correction was made in 7 patients, Urogynecologic and Vaginal Surgery Unit of Gynecology and Obstetrics Department, Las Condes Clinic. Medium age 54 years old, weight 64 kgs. In four patients we used a third mesh for correction of urinary incontinence by TVT-O.

Results: They don't present intraoperative complications, neither in immediate or delayed postoperative time. We not observed hematoma, infection, erosion or exposition mesh. Cure of cystocele and rectocele was in the $100 \%$ of patients. Complications have not appeared, 4 cases completed three months of observation, 2 cases two months and 1 case one month.

Discussion: The use of protesic polypropylene multifilament macropore mesh in the concomitant correction of cistocele and rectocele, by transvaginal route with tension free technique seems to be a safe and effective surgery procedure.

Keywords: Pelvic organ prolapse (POP) repair. Cystocele. Rectocele. Polypropylene mesh. Tension free. 
$\mathrm{L}$ as mallas de polipropileno han sido ampliamente utilizadas para reparación quirúrgica de defectos de pared abdominal. Es el caso de las hernias inguinales, y sobre todo las incisionales, entre otras. De esta forma se refuerza el defecto, disminuyendo la posibilidad de recidivas posteriores. Con el mismo principio de reforzar paredes, se han utilizado en ginecología, específicamente en la reparación de prolapsos como: cistocele, rectocele y suspensión de cúpula vaginal. De esta forma se podría disminuir la recurrencia, ya que con técnicas tradicionales fluctúa entre 5 y $40 \%{ }^{1}$. En los últimos años se han confeccionado mallas más flexibles y de mayor maleabilidad, tal es el caso de las mallas de monofilamentos de polipropileno.

Otro factor de gran importancia al escoger el tipo de malla a utilizar, es el tamaño del poro. El cual es primordial en la respuesta inflamatoria y reducción del riesgo de infección ${ }^{2,3}$.

La vía de corrección de estos defectos del piso pélvico puede ser vaginal o abdominal. También se han descrito técnicas laparoscópicas.

A continuación presentamos nuestra experiencia en la reparación del piso pélvico con malla de polipropileno monofilamento, por vía vaginal y con técnica libre de tensión.

\section{PACIENTE Y MÉTODO}

Durante el año 2004 se realizó corrección con malla de polipropileno monofilamento, de cistocele y rectocele concomitantemente en 7 pacientes, de la Unidad de Uroginecología y Cirugía Vaginal del Departamento de Ginecología y Obstetricia, de la Clinica Las Condes.

La mediana de edad fue de 54 años, peso $64 \mathrm{~kg}$.

Se utilizó malla Gynemesh PS (prótesis sintética no reabsorvible) con técnica libre de tensión transvaginal.

Cuatro pacientes además presentaban incontinencia de orina de esfuerzo, por lo que se agregó corrección con una tercera malla de polipropileno, por técnica de TVT-O (cinta libre de tensión transvaginal a agujero obturador).

Se utilizó profilaxis antibiótica durante la cirugía con una dosis simple de cefazolina $2 \mathrm{~g}$ intravenosa.
La analgesia utilizada fue valdecoxib $50 \mathrm{mg}$ vía oral una hora antes de la cirugía, repitiéndose monodosis oral cada 24 horas. Además ketoprofeno $100 \mathrm{mg}$ cada 8 horas endovenoso sólo el primer día (tres dosis).

A todas las pacientes se les explicó previamente, en qué consistía el procedimiento quirúrgico a realizar. Todas firmaron un consentimiento informado.

A todas se controló en consulta ambulatoria entre los 7 y 14 días, al mes, a los 3 y 6 meses. Además se mantuvo comunicación telefónica si era necesario.

\section{RESULTADOS}

Las pacientes presentaron cistocele grado $1 \mathrm{y}$ 2 , el rectocele era leve a moderado. Los 4 casos que presentaban incontinencia de esfuerzo correspondian a tipo II (según la clasificación urodinámica ICS 1999 preoperatoria).

Tiempo operatorio: La mediana de tiempo operatorio fue de 19 minutos para cistocele, y también 19 minutos para rectocele.

Complicaciones intraoperatorias: No se presentaron ni asociadas a la colocación de la malla en la corrección del cistocele, ni el rectocele. Tampoco hubo complicaciones durante el TVT. No se presentaron complicaciones respecto a la anestesia.

Complicaciones postoperatorias inmediatas (hasta los siete dias): No representaron complicaciones. No se observó hematomas ni infección de zona operatoria.

Analgesia: No fue necesario agregar farmacoterapia adicional al esquema para dolor postoperatorio planteado inicialmente. Ya que en todos los casos se logró un adecuado control, expresado por la paciente.

Levantada y alta hospitalaria:

Se levantó a todas las pacientes entre 12 y 24 horas. La alimentación se reinició entre 2 y 4 horas después de la cirugía.

El alta domiciliaria se dio a las 48 horas de operadas.

Complicaciones del postoperatorio tardio (mayor a una semana): No se han presentado a la fecha actual, completando 3 meses de observación 4 casos, 2 meses 2 casos, y 1 mes un caso. 


\section{DISCUSIÓN}

El primer estudio con mallas de polipropileno en la corrección del prolapso de pared anterior, fue realizado con Marlex en 1996, por Julian ${ }^{4}$. Se trataba de una randomización de 24 pacientes, 12 con corrección con malla. A los 24 meses se demostró una corrección anatómica en todas las pacientes con malla, versus un $66 \%$ en las con sólo colporrafia anterior. Migliari ${ }^{5}$ durante el 2000 demuestra en 12 pacientes a 20,5 meses de seguimiento, resultados de un $100 \%$ de curación en la corrección de cistocele con Prolene.

Actualmente no se debiera discutir el beneficio en disminuir la recurrencia de los prolapsos, al utilizar mallas protésicas de refuerzo tanto en defectos de pared vaginal anterior como posterior.

Sin embargo, al escoger una malla, debemos tener en cuenta el tamaño del poro, el cual, junto al material y trama de filamentos, otorga el grado de flexibilidad ${ }^{6}$. Por otro lado, debe tener un tamaño suficiente para permitir el paso de leucocitos $(9$ a $15 \mu)$ y macrófagos (16 a $20 \mu$ ) para reducir el riesgo de infección ${ }^{3}$. Las mallas de multifilamentos presentan espacios entre los filamentos de menos de $10 \mu$, permitiendo el pasaje de bacterias $(1 \mu)$, y no el de los macrófagos y leucocitos, facilitando la infección. Todo esto permite asegurar que la mejor malla sería monofilamento.

Las mallas de polipropileno corresponden a Tipo 1 según la clasificación de prótesis quirúrgicas ${ }^{7}$, es decir, monofilamento con macroporo $(75 \mu)$, cumpliendo con los requisitos mencionados anterior- mente. Por ello proponemos que debiera ser el gold Standard en la corrección con malla libre de tensión por vía vaginal, en la corrección de defectos del piso pélvico (Tabla 1). Clasificación de mallas sintéticas.

En otras publicaciones se ha descrito como principal complicación la erosión de pared vaginal con exposición de la malla ${ }^{8-11}$, principalmente en la corrección de cistocele, presentándose para Gynemesh entre $3,8 \%{ }^{10}$ y $7,5 \%{ }^{9}$. En las 7 pacientes que presentamos, no se ha demostrado esta complicación, ni en pared anterior, ni posterior vaginal.

Bader ${ }^{9}$ describió una duración de 20 minutos \pm 2,2 (16-24) en la técnica para cistocele, similar a el tiempo de nuestra experiencia. Sin embargo no existen publicaciones para el tiempo de duración de la técnica aplicada a rectocele.

La experiencia que comunicamos, se refiere a un reducido número de pacientes, sin embargo, se trata de casos con reparación concomitante de cistocele y rectocele, existiendo escasas publicaciones con estas características ${ }^{10}$. En otras publicaciones con corrección de malla con polipropileno monofilamento, macroporo, Gynemesh, se hace referencia a cistoscele exclusivamente ${ }^{8,9}$. La publicación de Adhoute ${ }^{10}$ comunica casos asociados o no a corrección con Gynemesh de cistocele y rectocele, con resultados efectivos de corrección a 27 meses de seguimiento, con 95\% para cistocele, y $100 \%$ para rectocele. En cambio Dweyer ${ }^{11}$ presenta casos de corrección de cistocele, asociados o no a rectocele con malla de polipropileno monofilamento Atrium (Hudson, New Hampshire, USA).

Tabla 1

Clasificación de mallas sintéticas.

\begin{tabular}{|c|c|c|c|c|}
\hline Tipo & Componente & Nombre comercial & Fibra & Tamaño Poro \\
\hline \multirow[t]{6}{*}{ Tipo 1} & Polipropileno & Prolene & Monofilamento & Macroporo \\
\hline & & Marlex & Monofilamento & Macroporo \\
\hline & & Atrium & Monofilamento & Macroporo \\
\hline & & Gynemesh & Monofilamento & Macroporo \\
\hline & Polipropileno/Poliglactin 910 & Vypro & Multifilamento & Macroporo \\
\hline & Poliglactin 910 & Vycril & Multifilamento & Macroporo \\
\hline Tipo 2 & Politetrafluoroetileno & Goretex & Multifilamento & Macroporo \\
\hline Tipo 3 & Polietileno & Mersilene & Multifilamento & Micro/Macro \\
\hline Tipo 4 & Polietileno & Cellgard (sin uso en ginecologia) & Monofilamento & Submicroporo \\
\hline
\end{tabular}

Macroporo: poro mayor de 75 micrones

Microporo: poro menor de 75 micrones 
Además, destacamos 4 casos con tres mallas, es decir, un procedimiento de TVT-O asociado con técnica clásica para corrección de la incontinencia de esfuerzo, que en estos casos no significó aumento de complicaciones, ya que no se presentaron. De este forma, se demuestra que puede ser seguro e igualmente eficaz la asociación de mallas en la corrección de defectos del piso pélvico e incontinencia urinaria.

Sin embargo, deberá comunicarse más adelante el seguimiento a largo plazo, además de continuar sumando pacientes con esta técnica combinada (2 o 3 mallas), para lo cual estamos realizando un seguimiento prospectivo.

\section{REFERENCIAS}

1. Lucente V, Hale D, Miller D, Madigan J. Oral Poster 55: A Clinical Assesment of Gynemesh PS for the repair of pelvis organ prolapse (POP). Journal of pelvic medicine \& Surgery. 2004; 10 Supplement 1:S 35.

2. Deval B, Haab F. What's new in preolapse surgery?. Curr Opin Urol 2003 Jul;13(4):315-323.

3. Brun JL, Bordenave L, Lefebre F, Bareille R, Barbie C, Rouais C, et al. Physical and biological characteristics of the main biomaterials used in pelvic surgery. Biomed Mater Eng 1992 Winter, 2(4):203-225.

4. Julian TM. The efficacy of Marlex mesh in the repair of severe, recurrent vaginal prolapse of the anterior midvaginal wall. Am J Obstet Gynecol 1996;175:1472-1475.
5. Migliari R, De Angelis M, Madeddu G, Verdacchi T. Tension free vaginal mesh repair for anterior vaginal prolapse. Eur Urol 2000;38:151-155.

6. Chu CC, Welch L. Characterization of morphologic and mechanical properties of surgical mesh fabrics. J Biomed Res 1985 Oct; 19(8):903-916.

7. Birch C, Fynes MM. The role of synthetic and biological prothesis in reconstructive pelvic floor surgery. Curr Opin Gynecol 2002;14:527-535.

8. De Tayrac R, Gervaise A, Fernández H. Cystocele repair by the vaginal route with a tension-free sub-bladdeer prothesis. J Gynecol Obstet Biol Reprod (Paris). 2002 Oct;31(6): 597-599.

9. Bader G, Fauconnier A, Roger N, Heitz D, Ville Y. Cystocele reapair by vaginal approach with a tension-free transversal polypropylene mesh. Technique and results. Gynecol Obstet Fertil 2004 Apr;32(4):280-284.

10. Adhoute F, Soyeur L, Pariente JL, Le Guillou M, Ferrier JM. Use of transvaginal polypropylene mesh (Gynemesh) for the treatment of pelvic floor disorders in women. Prospective study in 52 patients. Prog Urol 2004 Apr;14(2):192-196.

11. Dwyer Pl, O'Reilly BA. Transvaginal repair of anterior and posterior compartiment prolapse with Atrium polypropylene mesh. BJOG 2004 Aug;111(8):831-836.

Dr. P. Ricci Arriola

Unidad de Uroginecología y Cirugía Vaginal

Clinica Las Condes

Lo Fontecilla, 441 Las Condes

Santiago, Chile

E-mail: pricci@vtr.net

(Trabajo recibido el 17 de febrero 2005) 